\title{
Use of EBSD Data in Mesoscale Numerical Analyses
}

\author{
R. Becker, H. Weiland
}

This article was submitted to

The Minerals, Metals, and Materials Society, St. Louis, MO, October 8-12, 2000

\section{March 30, 2000}

\section{U.S. Department of Energy}

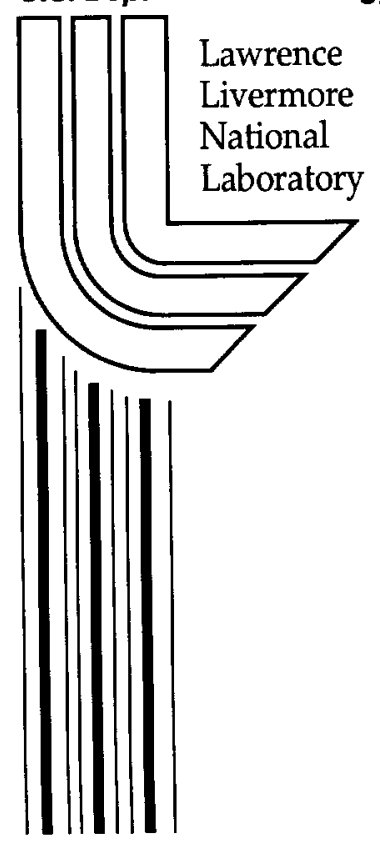




\section{DISCLAIMER}

This document was prepared as an account of work sponsored by an agency of the United States Government. Neither the United States Government nor the University of California nor any of their employees, makes any warranty, express or implied, or assumes any legal liability or responsibility for the accuracy, completeness, or usefulness of any information, apparatus, product, or process disclosed, or represents that its use would not infringe privately owned rights. Reference herein to any specific commercial product, process, or service by trade name, trademark, manufacturer, or otherwise, does not necessarily constitute or imply its endorsement, recommendation, or favoring by the United States Government or the University of California. The views and opinions of authors expressed herein do not necessarily state or reflect those of the United States Government or the University of California, and shall not be used for advertising or product endorsement purposes.

This is a preprint of a paper intended for publication in a journal or proceedings. Since changes may be made before publication, this preprint is made available with the understanding that it will not be cited or reproduced without the permission of the author.

This report has been reproduced directly from the best available copy.

Available electronically at http://www.doe.gov/bridge

Available for a processing fee to U.S. Department of Energy

and its contractors in paper from

U.S. Department of Energy

Office of Scientific and Technical Information

P.O. Box 62

Oak Ridge, TN 37831-0062

Telephone: (865) 576-8401

Facsimile: (865) 576-5728

E-mail: reports@adonis.osti.gov

Available for the sale to the public from

U.S. Department of Commerce

National Technical Information Service

5285 Port Royal Road

Springfield, VA 22161

Telephone: (800) 553-6847

Facsimile: (703) 605-6900

E-mail: orders@ntis.fedworld.gov

Online ordering: http://www.ntis.gov/ordering.htm

OR

Lawrence Livermore National Laboratory

Technical Information Department's Digital Library

http://www.llnl.gov/tid/Library.html 


\title{
USE OF EBSD DATA IN MESOSCALE NUMERICAL ANALYSES
}

\author{
Richard Becker ${ }^{1}$ and Hasso Weiland ${ }^{2}$ \\ ${ }^{1}$ Lawrence Livermore National Laboratory \\ Livermore, CA 94550 \\ ${ }^{2}$ Alcoa Technical Center \\ Alcoa Center, PA 15069
}

\section{INTRODUCTION}

Experimentation, theory, and modeling have all played vital roles in defining what is known about microstructural evolution and the effects of microstructure on material properties. Recently, technology has become an enabling factor, allowing significant advances to be made on several fronts. Experimental evidence of crystallographic slip and the basic theory of crystal plasticity were established in the early 20th century (Polanyi, 1922; Schmid, 1924; Taylor and Elam, 1925), and the theory and models evolved incrementally over the next 60 years (Taylor, 1938; Bishop and Hill, 1951; Hutchinson, 1964; Hill and Rice, 1972; Honneff and Mecking, 1978; Asaro, 1983a; Kocks et al., 1986). During this time, modeling was primarily concerned with the average response of polycrystalline aggregates. While some detailed finite element modeling (FEM) with crystal plasticity constitutive relations was performed in the early 1980's (Peirce et al., 1982,1983 ) such simulations over taxed the capacity of the available computer hardware. Advances in computer capabilities led to a flurry of activity in finite element modeling in the next 10 years (Harren et al., 1988; Havileck et al., 1990; Zikry and Nemat-Nasser, 1990; Becker et al., 1991; Kalidindi et al., 1992; Beaudoin et al., 1993; Saeedvafa and Rice, 1992; Mohan et al., 1992), thus increasing understanding of lattice orientation evolution and generating detailed predictions of spatial orientation distributions that could not be readily validated with existing experimental characterization methods.

Significant advancements in material characterization, particularly automated electron backscatter diffraction (EBSD), have made it possible to conduct detailed validation studies of the FEM predictions. The data collected are extensive, and many questions about the evolution of microstructure and its role in determining mechanical properties can now be addressed. It is now possible to obtain a detailed map of lattice orientations on a fine size scale. This will allow detailed quantitative comparisons of experiments and newly emerging large scale continuum FEM simulations. This capability will facilitate model validation efforts aimed at predicting deformation induced structural features, such as shear 
bands and cell structures, as well as predictions of the effects of grain interactions. The insight gained from the coupling of EBSD and FEM studies will provide impetus for further development of microstructure models and theories of microstructure evolution.

Early studies connecting EBSD data to detailed finite element models used manual measurements to define initial orientations for the simulations (Becker, 1991, 1998; Beaudoin et al., 1998). In one study, manual measurements of the deformed structure were also obtained for comparison with the model predictions (Becker and Panchanadeeswaran, 1995). More recent work has taken advantage of automated data collection on deformed specimens as a means of collecting detailed and spatially correlated data for FEM model validation (Weiland and Becker, 1999; Bhattacharyya et al., 2000).

Although it will not be discussed here, EBSD data can also be incorporated in FEM analyses in a less direct manner that is suitable for simulations where the element size is much larger than the grain size. The purpose of such models is to account for the effects of evolving material anisotropy in macro-scale simulations. In these analyses, a polycrystal plasticity model (e.g., a Taylor model or a self-consistent model), or a yield surface constructed from a polycrystal plasticity model, is used to determine the constitutive response of each element. The initial orientations used in the polycrystal plasticity model can be obtained from EBSD analyses (Bingert et al., Chapter 18, this volume) or by fitting distributions of discrete orientations to x-ray data (Becker, 1992; Kalidindi et al., 1992). The use of EBSD data is advantageous in that it is easier to account for spatial gradients of orientation distribution within a part.

Another area in which EBSD data is having a great impact is on recrystallization modeling. EBSD techniques can be used to collect data for quantitative microstructural analysis (Humphreys, 1998). This data can be used to infer growth kinetics of specific orientations, and this information can be synthesized into more accurate grain growth or recrystallization models (Vogel et al., 1996). A second role which EBSD techniques may play in recrystallization modeling is in determining initial structures for the models. A realistic starting structure is vital for evaluating the models, and attempts at predicting realistic structures with finite element simulations are not yet successful (Humphreys, 1999). As methodologies and equipment resolution continue to improve, it is possible that measured structures will serve as input for recrystallization models. Simulations have already been run using information obtained manually from a TEM (Radhakrishnan and Baggathun, 2000).

\section{CRYSTAL PLASTICITY MODEL}

The impetus behind the growing use of EBSD for characterization and analysis of orientation microstructures is the prominent role that crystal lattice orientations have in determining many material properties. This implies a corresponding need to include crystal orientation in material models. To construct models that can capture microstructural effects and that are truly representative of the material at grain level, it is important to incorporate the basic physical mechanisms. For deformation models, this means accounting for changes of shape and lattice orientation based on crystallographic slip. EBSD data provides a means to assess the strengths and weaknesses of these models so that their range of validity can be defined and areas for improvement can be identified.

The ideas of slip and lattice rotation were introduced in Taylor's (1938) seminal work, and they were given a more complete theoretical treatment in several later papers (e.g., Hill and Rice, 1972; Asaro, 1983b). Asaro (1983b) provides a comprehensive description of the basis for the crystal plasticity model. The concepts are generally accepted and serve as the basis for polycrystal texture evolution models such as the Taylor model, the Sachs 
model, and self-consistent models. Crystal plasticity constitutive relations have been incorporated into finite element codes for examining details of deformation in single crystals (Peirce et al., 1982, 1983; Harren et al., 1988; Becker et al., 1991; Saeedvafa and Rice, 1992; Mohan et al., 1992) and polycrystals (Havileck et al., 1990; Kaladindi et al., 1992; Becker, 1991, 1998; Beaudoin et al., 1998; Becker and Panchanadeeswaran, 1995). Although there have been many implementations of slip-based models, the basic kinematic description for most existing models is the same.

The integration methods used with early finite element implementations of crystal plasticity relations were developed for stepwise integration of the stress and state variables (Peirce et al., 1982, 1983; Harren et al., 1988; Havilcek, 1990; Zikry and Nemat-Nasser, 1990; Becker et al., 1991). Some more recent implementations (Kalidindi et al., 1992; Beaudoin et al., 1993; Miehe, 1996; Ortiz and Stainier, 1999; Harren, 2000) follow a total Lagrange formulation where the stress is evaluated directly from a strain energy function and arbitrarily large rigid body rotations can be accommodated within an increment. The kinematics are the same; it is the integration strategy which differs.

\section{Crystal Kinematics}

The basis for the kinematic description is a multiplicative decomposition of the deformation gradient, $\mathbf{F}$, into an elastic part, $\mathbf{F}^{*}$, and a plastic part, $\mathbf{F}^{P}$.

$$
\mathbf{F}=\mathbf{F}^{*} \cdot \mathbf{F}^{p}
$$

This is shown schematically in Figure 16.1. The plastic part captures the deformation by crystallographic slip. It does not distort the lattice or change its orientation. The elastic part accounts for rotation and distortion of the crystal lattice, and it provides the connection to EBSD data. Through the polar decomposition theorem, it can be written as

$$
\mathbf{F}^{*}=\mathbf{R}^{*} \cdot \mathbf{U}^{*}
$$

where $\mathbf{R}^{*}$ is the rotation taking the crystal from its reference orientation to its present orientation. This corresponds directly to the orientation obtained from an EBSD
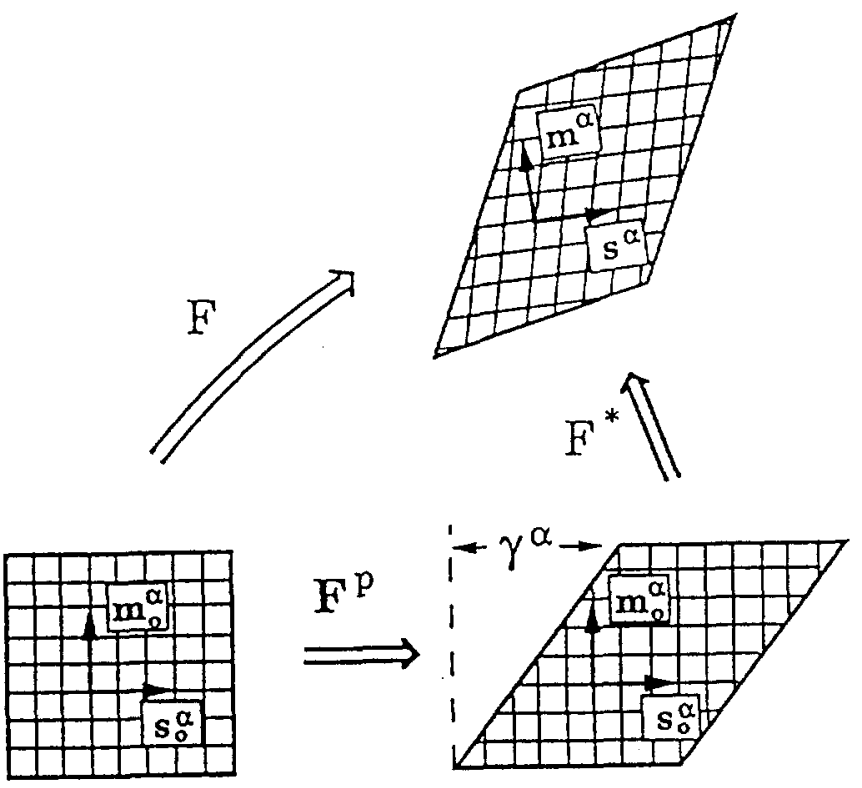

Figure 16.1. Illustration of deformation by slip and distortion of the crystal lattice. 
measurement. $\mathbf{U}^{*}$ represents the elastic distortion of the crystal lattice, which is small for typical metal forming problems. It is initially set to the identity for problems with no residual stress. In this case the orientations measured with EBSD can be input directly as the initial value of $\mathbf{F}^{*}$.

The plastic deformation within a volume element is specified in terms of the average slip rate, $\dot{\gamma}^{\alpha}$, on a slip system. The slip rate is related to the plastic part of the deformation gradient by

$$
\dot{\mathbf{F}}^{p} \cdot \mathbf{F}^{p-1}=\sum_{\alpha=1}^{N} \dot{\gamma}^{\alpha} \mathbf{s}_{\mathbf{0}}^{\alpha} \otimes \mathbf{m}_{0}^{\alpha}
$$

where the slip plane normal is given by $\mathbf{m}_{0}^{\alpha}$ and the slip direction is $\mathbf{s}_{0}^{\alpha}$. The superscript $\alpha$ is an index representing one of $N$ slip systems. Equation 16.3 is a representation of the deformation rate in the intermediate configuration of Figure 16.1, where the lattice is undistorted and in its initial orientation.

An expression giving the slip rate in the current configuration can be found by constructing the velocity gradient, $\mathbf{L}$, from Equation 16.1

$$
\frac{\partial \dot{\mathbf{x}}}{\partial \mathbf{x}}=\mathbf{L}=\dot{\mathbf{F}} \cdot \mathbf{F}^{-1}=\dot{\mathbf{F}}^{*} \cdot \mathbf{F}^{*-1}+\mathbf{F}^{*} \cdot \dot{\mathbf{F}}^{P} \cdot \mathbf{F}^{P-1} \cdot \mathbf{F}^{*-1}
$$

The first and second terms on the right hand side are the elastic and plastic parts of the velocity gradient, $\mathbf{L}^{*}$ and $\mathbf{L}^{p}$, respectively. Using Equation 16.3 the plastic part of the velocity gradient can be written as

$$
\mathbf{L}^{p}=\sum_{\alpha=1}^{N} \dot{\gamma}^{\alpha} \mathbf{s}^{\alpha} \otimes \mathbf{m}^{\alpha}
$$

where

$$
\mathbf{s}^{\alpha}=\mathbf{F}^{*} \cdot \mathbf{s}_{0}^{\alpha} \quad \text { and } \quad \mathbf{m}^{\alpha}=\mathbf{m}_{0}^{\alpha} \cdot \mathbf{F}^{*-1}
$$

are the slip direction and slip plane normal in the current configuration. $\mathbf{s}^{\alpha}$ and $\mathbf{m}^{\alpha}$ are assumed to be orthogonal for slip processes, so the plastic part of the deformation is isochoric.

Equations can be written for the evolution of $\mathbf{s}^{\alpha}$ and $\mathbf{m}^{\alpha}$ in terms of $\dot{\mathbf{F}}^{*}$ by taking time derivative of Equation 16.6. However, in practice they are typically evaluated directly from Equation 16.6 for a given $\mathbf{F}^{*} . \mathbf{F}^{*}$ is either integrated from

$$
\dot{\mathbf{F}}^{*}=\left(\mathbf{L}-\mathbf{L}^{p}\right) \cdot \mathbf{F}^{*}
$$

or it is calculated through Equation 16.1 where $\mathbf{F}$ is known from the configuration and $\mathbf{F}^{P}$ is integrated from Equation 16.3

$$
\dot{\mathbf{F}}^{p}=\left(\sum_{\alpha=1}^{N} \dot{\gamma}^{\alpha} \mathbf{s}_{\mathbf{0}}^{\alpha} \otimes \mathbf{m}_{\mathbf{0}}^{\alpha}\right) \cdot \mathbf{F}^{p}
$$

Integration through Equation 16.7 is used with updated Lagrangian methods, while the combination of Equations 16.1 and 16.8 is often used in full Lagrangian formulations. 


\section{Stress-Strain Relations}

It is assumed that the stress is related to the elastic distortion of the crystal lattice through a strain energy function $\phi$. The second Piola-Kirchhoff stress, $T^{*}$, and its rate can be given by

$$
\mathbf{T}^{*}=\frac{\partial \phi}{\partial \mathbf{E}^{*}} \quad \text { and } \quad \dot{\mathbf{T}}^{*}=\frac{\partial^{2} \phi}{\partial \mathbf{E}^{* 2}}: \dot{\mathbf{E}}^{*}=\mathcal{K}: \dot{\mathbf{E}}^{*}
$$

The Lagrangian strain of the lattice, $\mathbf{E}^{*}$, is

$$
\mathbf{E}^{*}=\frac{1}{2}\left(\mathbf{C}^{*}-\mathbf{I}\right) ; \quad \mathbf{C}^{*}=\mathbf{F}^{* T} \cdot \mathbf{F}^{*}
$$

where $\mathbf{C}^{*}$ is the right Cauchy-Green stretch tensor of the lattice. $\mathcal{K}$ in Equation $16.9 \mathrm{~b}$ is the fourth order crystal modulus tensor, usually set in a reference frame coincident with principle lattice directions.

The second Piola-Kirchhoff stress is related to the Kirchhoff stress, $\tau$, and the Cauchy stress, $\sigma$, as

$$
J \sigma=\tau=\mathbf{F}^{*} \cdot \mathbf{T}^{*} \cdot \mathbf{F}^{*}
$$

where $J=\operatorname{det}|\mathbf{F}|$, is the ratio of the deformed volume to the reference volume. Since the plastic deformation is isochoric, $J=\operatorname{det}\left|\mathbf{F}^{*}\right|$, the elastic volume change. $J$ is typically very close to one for industrial forming operations, so the distinction between the Cauchy stress and the Kirchhoff stress is usually ignored.

An expression for the stress rate can be obtained by taking the derivative of Equation 16.11 and combining terms (Asaro, 1983). After some manipulation, the Jaumann rate of the Kirchhoff stress can be written as

$$
\hat{\tau}=\dot{\tau}-\omega \cdot \tau+\tau \cdot \omega=\mathcal{M}: \mathbf{D}-\sum_{\alpha=1}^{N} \dot{\gamma}^{\alpha} \mathbf{R}^{\alpha}
$$

where $\mathbf{D}$ is the rate of deformation tensor (symmetric part of the velocity gradient); $\omega$ is the spin tensor (asymmetric part of the velocity gradient); $\mathcal{M}$ is a fourth order modulus obtained by rotating the crystal modulus $\mathcal{K}$ into the laboratory reference frame and adding a few terms on the order of stress; $\mathbf{R}^{\alpha}$ is given by

$$
\mathbf{R}^{\alpha}=\mathcal{M}: \mathbf{P}^{\alpha}+\mathbf{W}^{\alpha} \cdot \tau-\tau \cdot \mathbf{W}^{\alpha}
$$

and

$$
\mathbf{P}^{\alpha}=\frac{1}{2}\left(\mathbf{s}^{\alpha} \otimes \mathbf{m}^{\alpha}+\mathbf{m}^{\alpha} \otimes \mathbf{s}^{\alpha}\right) \quad \text { and } \quad \mathbf{W}^{\alpha}=\frac{1}{2}\left(\mathbf{s}^{\alpha} \otimes \mathbf{m}^{\alpha}-\mathbf{m}^{\alpha} \otimes \mathbf{s}^{\alpha}\right)
$$

Equation 16.12 is used in updated Lagrangian integration schemes where the stress is integrated in time. Equation $16.9 \mathrm{a}$ is often used to compute the stress directly from $\mathbf{E}^{*}$ in full Lagrangian formulations. Although the relations for the full Lagrangian approach appear much simpler, the derivatives needed for a Newton iteration scheme resemble Equations 16.12 and 16.13. Actual coding for the full Lagrangian formulation can be somewhat more involved because of convergence checking and correction updates. The advantage of the full Lagrange formulation is stability and accuracy for large rigid body rotation increments. 


\section{Slip System Constitutive Relations}

For crystals obeying the Schmid criterion, slip is assumed to be related only to the resolved shear stress on the slip systems. In a rate independent model, the slip rate is generally taken to be proportional to the rate of change of the resolved shear stress. In a rate dependent model, the slip rate is assumed to be related to the resolved shear stress itself, not its rate. The resolved shear stress on a slip system, $\tau^{\alpha}$, can be determined from

$$
\tau^{\alpha}=\mathbf{m}_{0}^{\alpha} \cdot \mathbf{T}^{*} \cdot \mathbf{C}^{*} \cdot \mathbf{s}_{0}^{\alpha}
$$

in the intermediate configuration of Figure 16.1, and

$$
\tau^{\alpha}=\mathbf{m}^{\alpha} \cdot \tau \cdot \mathbf{s}^{\alpha}
$$

for a formulation based in the current configuration.

Because slip on multiple combinations of slip systems can accommodate many deformation modes, rate independent constitutive models can lead to ambiguities in the solution. The degree of slip on each system may be indeterminate. Although use of some slip system hardening relations (Bassani, 1990) or the single valued decomposition method (Anand and Kothari, 1996) can be used to obtain a solution, the most common method currently employed to circumvent this slip system indeterminacy is to use a rate dependent slip system model. Here the slip rate is given uniquely in terms of the resolved shear stress on the slip system, $\tau^{\alpha}$, and its resistance to slip, $g^{\alpha}$.

A simple power law strain rate sensitivity takes the form:

$$
\dot{\gamma}^{\alpha}=a \operatorname{sign}\left(\tau^{\alpha}\right)\left(\left|\frac{\tau^{\alpha}}{g^{\alpha}}\right|\right)^{1 / m}
$$

where $a$ is a reference slip rate and $m$ is the strain rate sensitivity exponent.

The resistance of a slip system can be written as an evolution equation

$$
\dot{g}^{\alpha}=\sum_{\beta=1}^{N} H_{\alpha \beta} \dot{\gamma}^{\beta}
$$

where $H_{\alpha \beta}$ is an evolving matrix capturing hardening interactions among slip systems. The model allows considerable flexibility for specifying the strength evolution, but most current simulations adopt a rather simple specification where $H_{\alpha \beta}$ evolves proportionally with deformation:

$$
H_{\alpha \beta}=g^{\prime} h_{\alpha \beta}
$$

Here, $g^{\prime}$ is typically assumed to be a function of the accumulated slip with $h_{\alpha \beta}$ being a constant matrix. If the hardening of a slip system due to its own activity is assumed to be equal to the hardening due to activity on other slip systems, $h_{\alpha \beta}$ is fully populated with ones. This is the most common assumption, and it was the assumption used in generating the results presented here. More comprehensive slip system hardening models have been proposed that more accurately represent detailed observations of secondary slip initiation (Bassani and $\mathrm{Wu}, 1991$; Cuitino and Ortiz, 1992), but these have not yet found widespread use in modeling activities. 


\section{CRYSTAL MODEL VALIDATION}

As the automated collection of EBSD data is relatively new, methods of coupling the experimental data to finite element simulations are still under development. While there have been several excursions into applications (Becker, 1998; Beaudoin et al., 1998), most detailed, joint EBSD-FEM studies to date have been conducted for purposes of model validation. In these studies, the orientations measured from EBSD are compared with those calculated from the finite element model either though pole figures or spatial maps of the orientation distribution. Some earlier studies used manually collected measurements of orientations on a section before and after deformation to assess the validity of FEM solutions (Becker and Panchanadeeswaran, 1995). More recent efforts are using automatically collected data and three-dimensional deformation models (Weiland and Becker, 1999; Bhattacharyya et al., 2000).

\section{Split Channel Die Polycrystal Sample}

An early validation study using EBSD data was conducted by Panchanadeeswaran et al., (1996). A cast commercial purity aluminum sample with a grain size of $200 \mu \mathrm{m}$ was machined for a channel die compression experiment. The sample was split in half with the dividing plane normal being in the constraint direction of the specimen. Both new surfaces were polished. One of the mating surfaces had a region marked with microhardness indents over a $2 \times 2 \mathrm{~mm}$ square area. The grain morphology and orientations in the region were then recorded, Figure $16.2 \mathrm{a}, \mathrm{b}$. The sample was reassembled and deformed to $40 \%$ reduction in the channel die at $375^{\circ} \mathrm{C}$.

The locations of the hardness indents were recorded from the deformed sample and used to calculate the average strain experienced by the region. There was substantial shear in addition to the compression, as evident in Figure 16.2c. This shear is consistent with the inadvertent macroscopic shear of the specimen during the compression test. Manual orientation measurements were made on several grains in the marked area of the deformed sample.

The measured locations of the initial grain boundaries from a region containing 35 grains were used to construct a two dimensional finite element mesh of 2187 quadrilateral elements in which the mesh lines conformed to the grain boundaries. The mesh was continuous across the grain boundaries, simulating deformation with no grain boundary sliding. The initial lattice orientations for each grain were assigned based on the measurements.

The slip system hardening and strain rate sensitivity used in the simulation were deduced by taking polycrystal data and using an average Taylor factor to scale the stress and strain rate to obtain approximate values of resolved shear stress and slip rate. All slip systems were assumed to harden equally. With this construction, a Taylor polycrystal model will approximately replicate the original experimental stress strain curve. The two dimensional model simulated deformation in plane strain compression with the final applied configuration being consistent with that measured from the microhardness indents, Figure 16.2c.

Comparison of the experimental and calculated orientation distributions for individual grains shows some for which the agreement is good, Figure 16.3a and others where the simulations failed to predict the observed orientation spread, Figure 16.3b. In general, agreement was only found when orientation changes were small. Since such grains do not provide a critical test for the model, one might be hesitant to conclude that the model predictions were proven successful for any of the grains examined. 


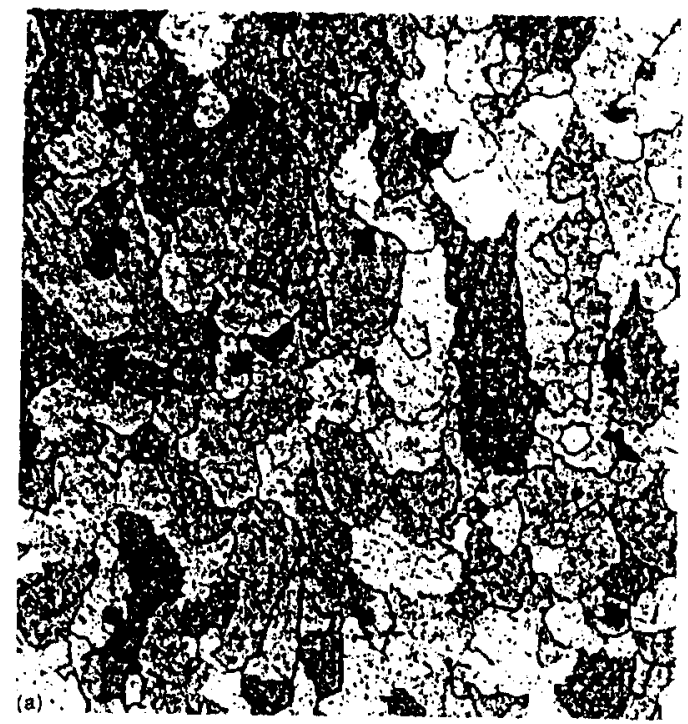

b)

b)

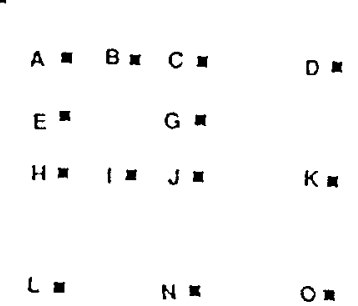

c)

Figure 16.2. Microhardness indents on the split channel die compression specimen: a) as seen on the specimen; b) initial indent pattern and c) pattern after deformation.

Observation of many pole figures from the simulation showed that orientation changes resulted primarily from rotations about an axis normal to the model plane. This was the case even though all twelve slip systems were modeled and the lattice was free to rotate about any arbitrary axis in three dimensional space. Rotation only about the model plane was also observed in an earlier study (Becker, 1991). This behavior is thought to be a result of the kinematic freedom for material spin about that axis and thus only captures shear stress and grain interactions in the model plane. The same grain configuration was used in another simulation with the initial orientations rotated 90 degrees about the extension axis. The deformation mode was generalized plane strain, with the compression being the out of plane direction. The coincidence of the rotation axis with the normal to the model plane was again evident (Becker and Panchanadeeswaran, 1995). This verified that the predominant rotation about the model plane is indeed an artifact of the twodimensional model. In addition to these difficulties, it was clear from the results that grain shapes and neighboring grains have a significant impact on orientation changes. Thus, it is important to include grain interactions in all directions.

A conclusion from the study was that it is not possible to obtain a realistic approximation of a deformed three dimensional microstructure with a two dimensional model except for a very restricted class of orientations. Accurate predictions of local lattice orientations require a detailed three dimensional representation of the microstructure and a means of applying boundary conditions to the model without introducing significant artificial constraint.

These conclusions suggest two paths for constructing model validation studies: characterize and test a three-dimensional grain structure or construct idealized specimens for which the grain structure is known. With the technology available until recently, the former option involved serial sectioning where the specimen is destroyed. This prohibits one to one validation, but it is useful for deformation studies on a larger size scale. Such characterization has been performed on a tantalum specimen (Stölken et al., 1999). Recent advances in 3-D characterization using synchrotron radiation look promising as a nondestructive method of mapping the initial microstructure (Juul Jensen, Chapter 8, this volume). This technology may enable 3-D validations studies in the near future. The second validation option is achievable by constructing columnar grain samples where the grain structure is constant in one direction. While the configuration is not representative of a typical polycrystal sample, it is useful for model validation. 

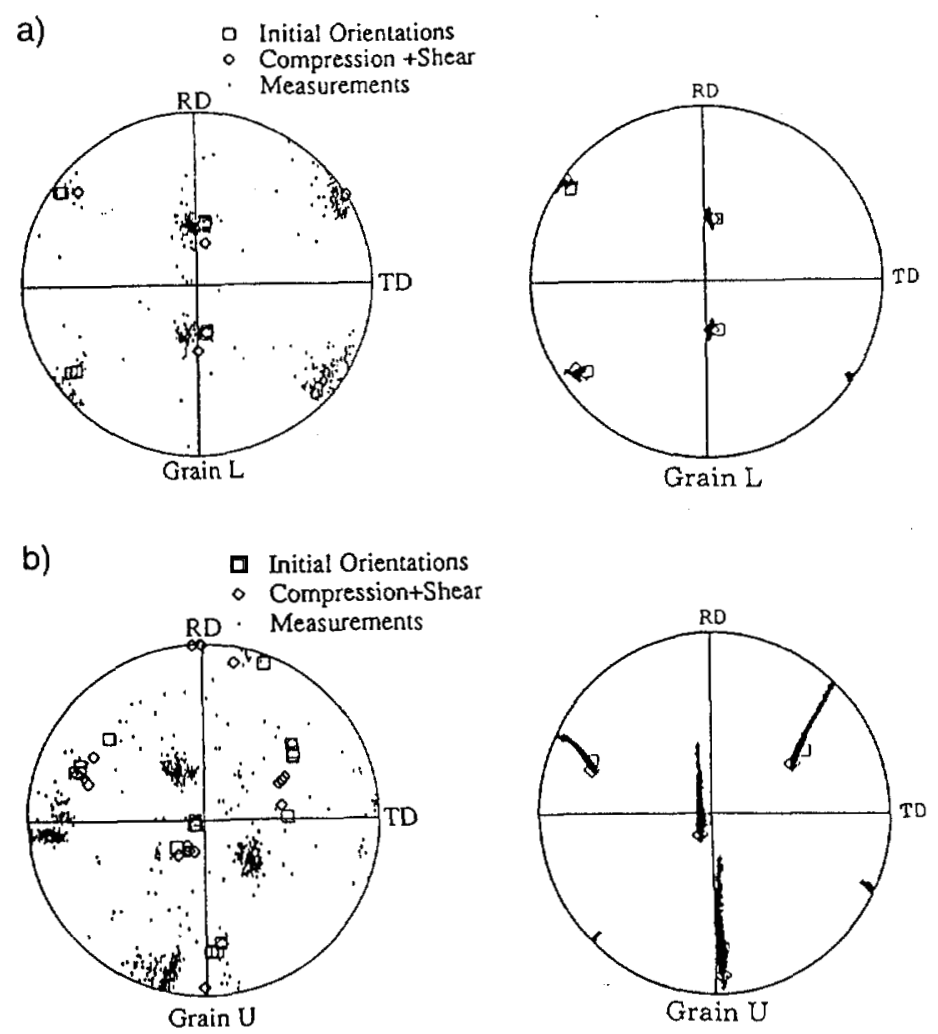

Figure 16.3. Comparison of experimental and predicted (111) pole figures from the split channel die compression study. Examples of a) agreement and b) disagreement. Experimental pole figures are on the left and the model results are on the right.

\section{Deformation of a Quasi-Columnar Grain Sample Along the Growth Direction}

Two recent studies (Weiland and Becker, 1999; Bhattacharyya et al., 2000) have used columnar grain samples in coordinated EBSD-FEM validation efforts. Both studies started with the same directionally solidified aluminum castings that produced quasi-columnar grains running in the solidification direction. Nearly all of the grains had a (001) crystal direction approximately aligned with the solidification direction. In the work by Bhattacharyya et al., (2000), a $3 \mathrm{~mm}$ thick slice of the casting was examined where the columnar axis was in the thickness direction of the slice. The sample was characterized by EBSD on both sides of the slice. Since the columnar grain growth was imperfect, there was a variation in grain shape from one side to the other. One side had coarser grains, indicating that some of the grains terminated within the specimen. The sample was compressed $40 \%$ of its initial height by uniaxial compression with the compression axis being the axis of the columnar grains. Since the initial orientations were near (001) and the stable orientation for uniaxial compression is (110), considerable lattice rotation was expected.

The deformed specimen was characterized by automated EBSD for comparison with the simulations. A prominent feature found on the orientation maps was the occurrence of distinct bands or regions of differing orientation within the grains. In some grains the bands were solitary, while in others, several narrow parallel bands of alternating orientation were observed.

A uniform finite element mesh of hexahedral elements was created to model the compression specimen. Mesh regions were identified with specific grains from the initial EBSD map of the coarse grained side of the sample, and the appropriate orientations were assigned to the elements. Since the mesh was regular, the mesh lines did not conform to 
the grain boundaries, and the grain boundaries in the model had a stepped appearance. The specimen was modeled with one element through the thickness of the slice. Boundary conditions applied to the four planes orthogonal to the compression plane required that these faces remain planar and orthogonal.

An orientation map constructed from the deformed finite element model failed to show the orientation banding observed in the experiments, and comparison of the predicted pole figures with the experimental results also failed to show the correct distribution of lattice rotation in many grains. These two observations are consistent and suggest that the local deformation pattern may not have been predicted correctly either. Since deformation is a combination of rotation and strain, this lack of agreement in orientation also suggests that the deformation pattern may be in error. Potential reasons for failure of the model will be discussed below.

\section{Transverse Deformation of a Quasi-Columnar Grain Sample}

In another study (Weiland and Becker, 1999) using the columnar grain material, segments of the casting were annealed prior to excising the specimens. This produced centimeter-sized grains with considerably less variation along the growth axis. Channel die compression specimens with dimensions $10 \mathrm{~mm}$ thick by $15 \mathrm{~mm}$ high by $25 \mathrm{~mm}$ long were machined for this study. The growth axis of the grains was aligned with the constraint direction (10 $\mathrm{mm}$ direction) of the channel die. The grain patterns were very similar on both sides of the specimen, indicating that the desired columnar grain structure was nearly achieved. The initial grain structure was photographed on all faces, and the grain orientations were measured at several points by EBSD. As with the study described above, the grains tended to have the (001) direction aligned within 5 to 10 degrees of the growth axis. The samples were deformed to $15 \%$ compression in a channel die at room temperature and at $200^{\circ} \mathrm{C}$.

Here, attention will be focused on one sample that contained three grains. The specimen and its initial lattice orientations are shown in Figure 16.4. The deformed specimen is shown in Figure 16.5. The labels CD and ED refer, respectively, to the compression direction and extension direction of the sample. As the sample was deformed in a channel die, little deformation occurred in the constraint direction. However, deformation by slip has normal-shear coupling, and the deformation on the plane normal to the constraint direction varied through the thickness of the sample. For example, the "foot" on the lower right of the deformed sample varied in size and shape through the thickness of the specimen.
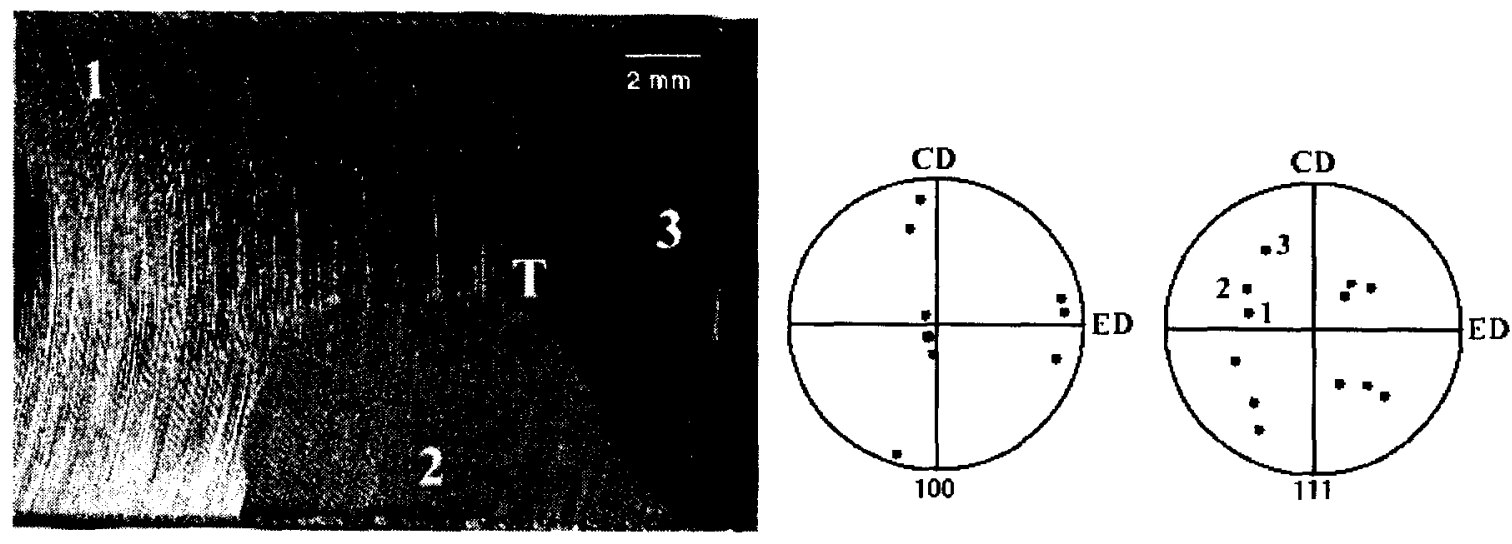

Figure 16.4. Initial specimen geometry and orientation for columnar grain tricrystal. 

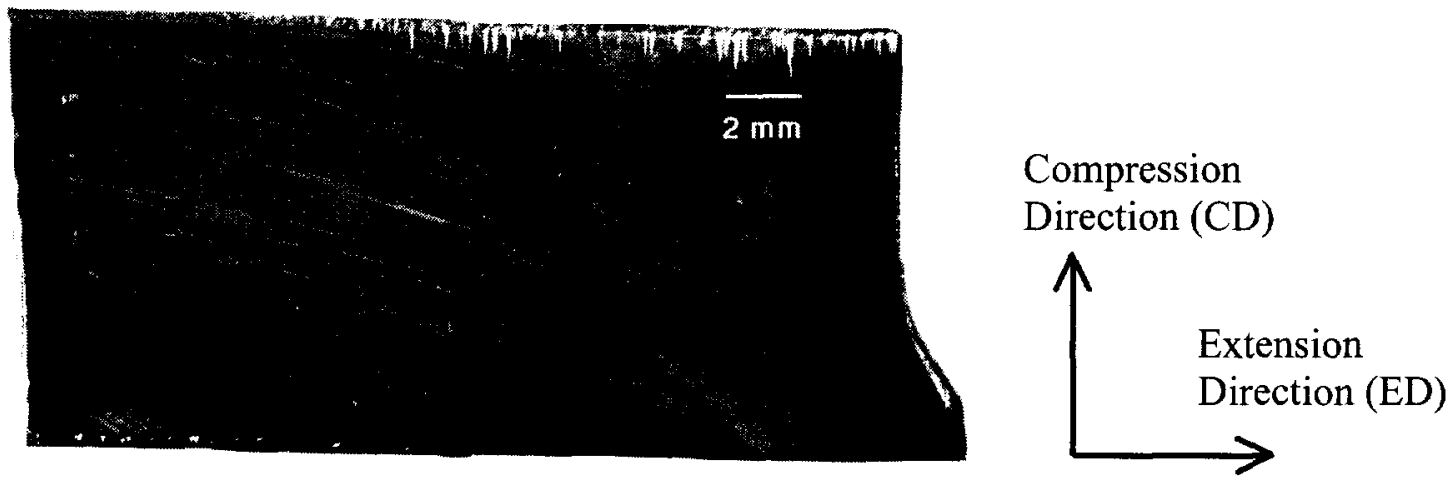

Figure 16.5. Columnar grain tricrystal following $14 \%$ compression in a channel die. The white rectangles mark regions where OIM scans were taken.

EBSD scans of the deformed sample were taken from within the three boxes indicated on Figure 16.5. Two of these are within single crystal regions fairly far removed from a grain boundary and the third, the center box, includes all three grains near the triple point. Orientation maps from these three regions and the corresponding (111) pole figures are shown in Figures 16.6-16.8. The contour maps in Figures 16.6 and 16.7 were created by selecting a location near the center of the region and applying the same shading to all points which had an orientation within 5 degrees of the selected orientation. Regions with an orientation greater than 5 degrees from the reference were shaded another color. The same shading is applied to the corresponding points on the pole figures. The white lines on the contour plots represent the orientations of slip traces observed on the surface of the deformed specimen.

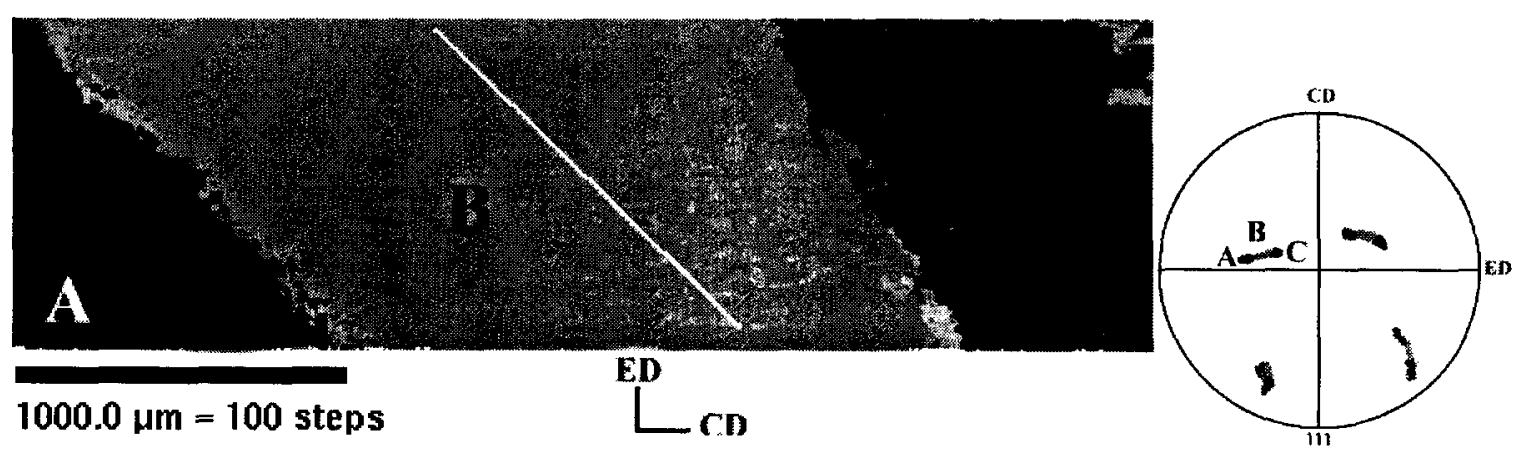

Figure 16.6. EBSD orientation map from the region in grain 1 of Figure 16.5. The white line indicates a slip trace.

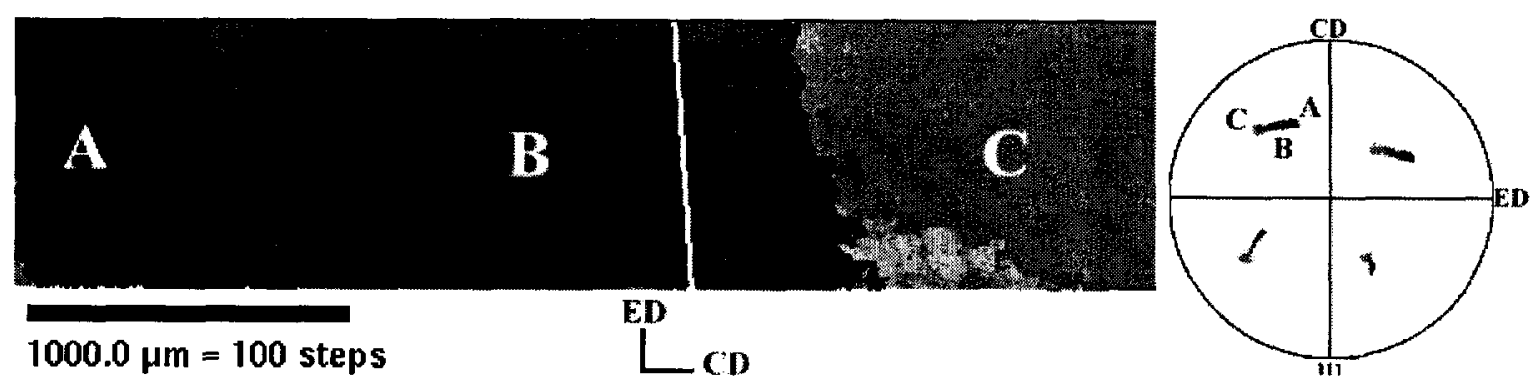

Figure 16.7. EBSD orientation map from the region in grain 3 of Figure 16.5. The white line indicates a slip trace. 

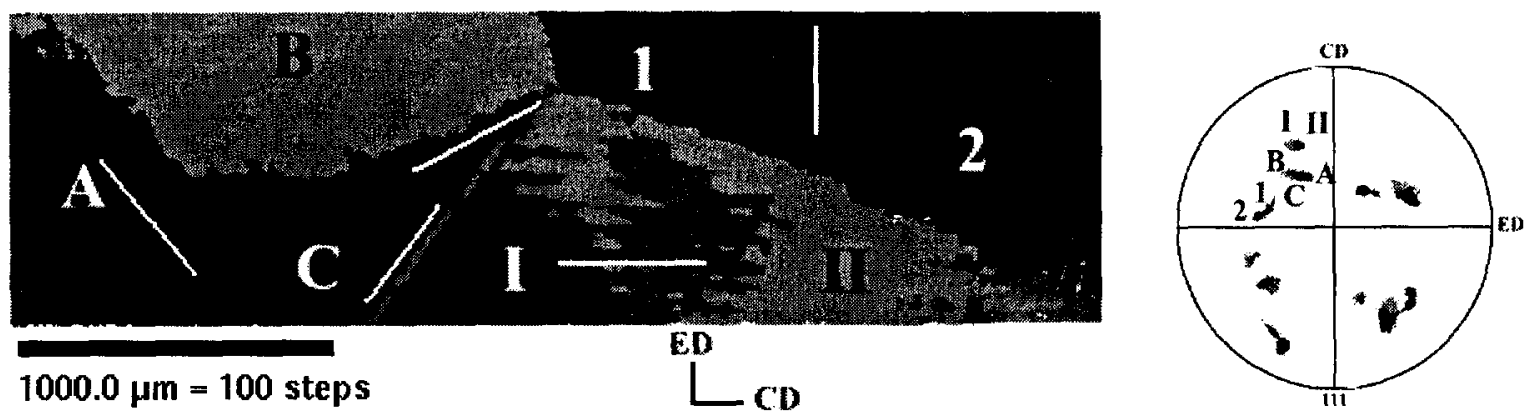

Figure 16.8. Orientation map covering the triple junction of the tricrystal. Regions labeled 1 and 2 are subdivisions of grain \#1; A, B and C are subdivisions of grain \#2; and I and II are subdivisions of grain \#3. The white line indicates the slip trace direction.

Figures 16.6 and 16.7 are, respectively, from the large single crystal regions on the left and right ends of the specimen. In both cases, the lattice rotation from one end of the plot to the other is greater than 10 degrees. The contour boundaries are aligned with the slip traces in both cases. This suggests that the orientation is roughly constant along the slip trace and that the orientation gradient is greatest in the direction normal to the slip plane. Had slip occurred uniformly in the region, the lattice would have the same orientation throughout. Hence, the plots indicate a significant gradient in slip activity over the region.

The EBSD scan containing the triple point, Figure 16.8, is more complex, but the same correlation can be observed between the orientation gradients and the dominant slip trace: the orientation is fairly constant along the direction of the slip trace, and it varies normal to the trace. It is also observed that the slip traces lie along different directions than in the regions of the crystal remote from the triple junction. This is a result of the complex stress state found at the triple point. Grain 3 in Figure 16.8 also displays a banded orientation structure reminiscent of that observed by Bhattacharyya et al. (2000). The lack of such a structure in other portions of the same grain, Figure 16.7, supports the hypothesis that such structures form for certain combinations of loading and crystal orientations but not others.

The finite element mesh used to simulate the deformation is shown in Figure 16.9. It contains approximately 20000 hexahedral elements. The lattice orientations were assigned based on the EBSD measurements. Boundary conditions were chosen to simulate deformation in a channel die. The width of the channel was wider than the undeformed specimen to permit insertion of the specimen along with PTFE tape for a lubricant. To capture the initial broadening of the specimen, the channel was modeled as rigid surfaces $10.35 \mathrm{~mm}$ apart while the initial specimen width was $9.98 \mathrm{~mm}$. This allows a lateral spread of approximately $4 \%$. Deformation was achieved by displacing the upper surface with respect to the lower at a constant velocity.

The deformed specimen shape and predicted contours of accumulated slip are shown in Figure 16.10. The specimen outline shows that a "foot" was predicted on the lower left of the specimen, but the magnitude and shape differ from that observed in Figure 16.5. Another feature of the deformed shape worth noting is the slope of the boundary on the left side of the crystal. The simulation predicts that the boundary will be inclined with the top moving toward the right with respect to the bottom. The experiment shows the top moving toward the left with respect to the bottom. This is obviously a source of concern. In attempts to obtain better qualitative agreement, many perturbations were made to the initial orientation, the friction, slip system hardening relations, strain rate sensitivity and channel width. None of these measures caused the slope on the left side of the model to have the 
correct sign. The predicted pole figure from the model region corresponding to Figure 16.6 shows no discernible orientation spread and very little lattice rotation. This is consistent with Figure 16.10, which shows little difference in accumulated slip across the region.

The reason that the models fails to predict several gross features of the experiment is not known. Two possible explanations that were not explored are: the finite element discretization may inhibit gradient development; and the coarse slip bands may alter the slip system hardening relations. These issues will be discussed in more detail later.

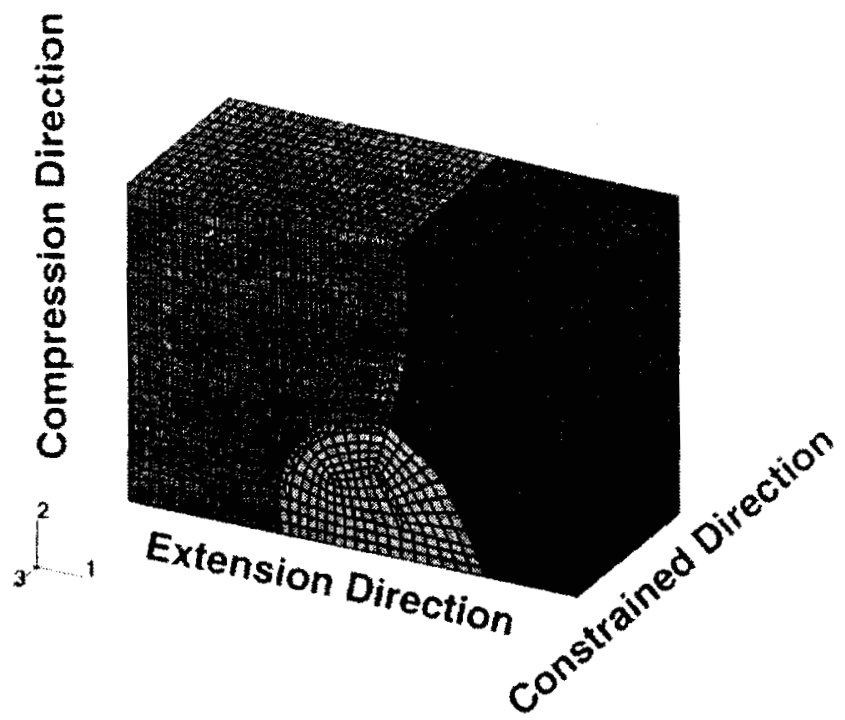

Figure 16.9. Finite element mesh used to simulate the deformation of the columnar grain tricrystal.

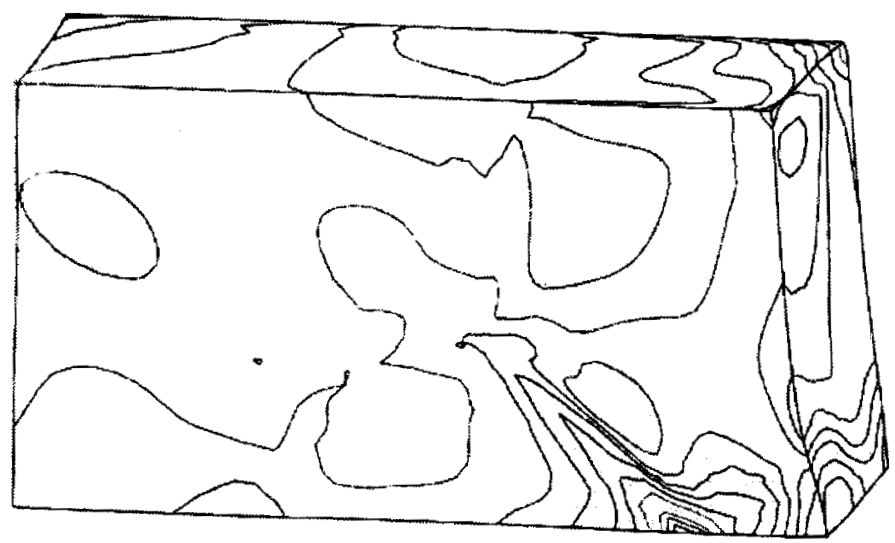

Figure 16.10. Predicted contours of accumulated slip from the finite element model of the tricrystal.

\section{Direct Mapping of EBSD Data to a Finite Element Simulation}

In the examples cited above, regions of the finite element model were defined as grains based on grain boundaries identified through micrographs or EBSD data. All of the elements within each grain were assigned the same initial orientation. A more direct and automated assignment of orientations was explored by Kallivayalil et al. (1998). In that 
work, a region near the surface of an aluminum sample was characterized by an automated EBSD scan. A $100 \times 250$ regular finite element mesh was constructed to be the same size as the scanned region. The initial lattice orientation of each element was determined by calculating the element centroid coordinates and locating the nearest corresponding point in the EBSD map. The orientation of this point in the EBSD database was computed in terms of a rotation matrix, and this matrix was used as the initial $\mathbf{F}^{*}$ in the model. As a result of this initialization procedure, any orientation variation present in the measurements was reflected in the finite element model. A contour plot of $F_{11}$, a component of the rotation matrix identifying the initial orientation of the crystal lattice, is shown in Figure 16.11. The plot looks remarkably like an EBSD orientation map.

The free surface of the specimen is at the bottom of the model shown in Figure 16.11. The nodes on the upper surface were constrained from motion in the vertical direction but were free to move horizontally. On the left side of the model, the nodes were constrained horizontally and free to move vertically. Deformation was applied by moving the nodes on the right side of the model. The region was deformed $4 \%$ in plane strain tension.

Contours of accumulated slip from the simulation are shown in Figure 16.12. The notable feature of these results is the appearance of fine structure and slip patterning. These features appear realistic but have not been observed in other simulations. They could be the result of a combination of factors, but further exploration is needed to determine a definite origin. The most obvious factors distinguishing this model from others are: i) a very fine spatial discretization giving the solution significantly more degrees of freedom, ii) a large number of grains and interacting neighbors to drive nonuniform deformation patterns, and iii) the presence of a free surface on the bottom of the model.
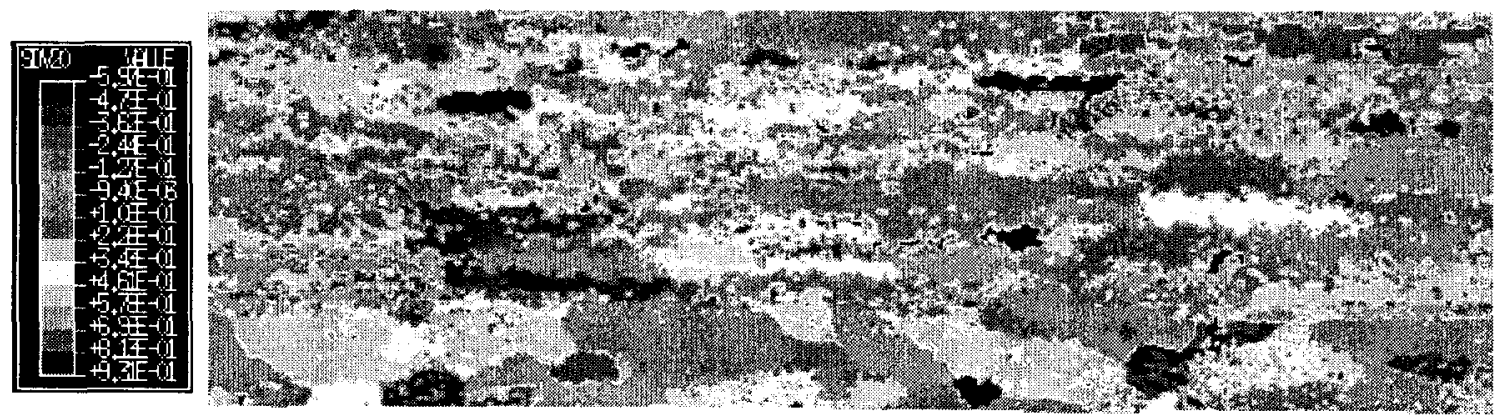

Figure 16.11. Contour plot from the FEM code showing the " 11 " component of the rotation matrix defining the initial lattice orientations.
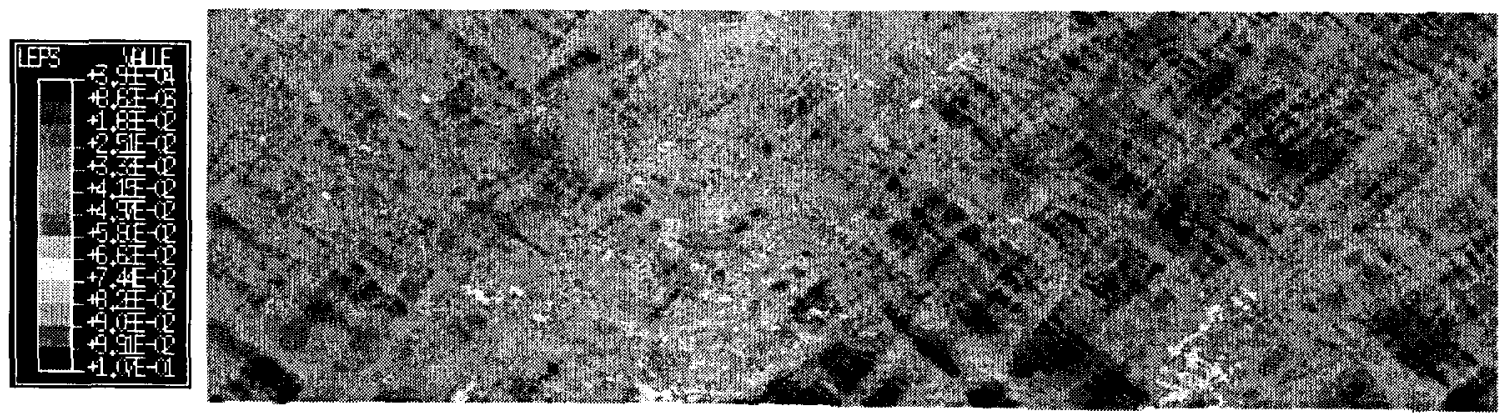

Figure 16.12. Contours of maximum principal logarithmic strain following $4 \%$ strain of the model with orientations defined in Figure 16.11. 


\section{CRYSTAL MODEL VALIDATION}

The availability of data collected from automated EBSD enables a critical evaluation of the accuracy of mesoscale finite element crystal plasticity models. The significant disagreement found between model and experiment in the examples presented above highlights the importance of conducting such validation studies. Validation studies define the expected quality of the predictions and identify aspects of the problem which must be defined accurately to obtain a reasonable solution.

Based on the success of Taylor-like polycrystal models for predicting texture evolution, it was at first anticipated that a discretized model of a two dimensional section would give nearly correct orientation predictions since equilibrium and compatibility could both be satisfied. This proved not to be the case. It was then suggested that properly accounting for the details of the grain structure and the boundary conditions would fix the problem. This was also not sufficient. Even when the full three dimensional grain structure was known and boundary condition approximations were avoided by modeling the full specimen, the model failed to predict gross features of the specimen deformation as well as the significant orientation gradients. There is obviously something else that is not being modeled properly.

The potential causes of the disagreement have not been investigated thoroughly, so additional studies must be conducted to isolate the factors and propose guidelines for future modeling. Possible sources of modeling error can be put into two broad classes: deficiencies in the finite element model and deficiencies in the crystal model itself.

Of several possible finite element model deficiencies, one is clearly inadequate spatial discretization. In the columnar grain studies of Bhattacharyya et al. (2000), the element size was larger than $150 \mu \mathrm{m}$ and the element size was on the order of $700 \mu \mathrm{m}$ in the study by Weiland and Becker (1999). In both cases, the experiments showed band formation with a spacing on the order of $100 \mu \mathrm{m}$. To capture features of this size, the elements would need to be on the order of $10 \mu \mathrm{m}$ or less. It is clear that the models would never capture these features with the given discretization. The element size must be much smaller than the size of the important features that are to be captured.

The finite element model will also not allow abrupt orientation changes unless the plane across which the orientation changes coincides with the mesh lines. Such abrupt orientation changes are prominent features near the triple point in Figure 16.8, and they are also found in EBSD studies of single crystals (e.g., Schwartz et al., 2000). If the mesh alignment prohibits the formation of these bands, the solution could be perturbed significantly.

Additionally, the alignment of the elements with the direction of the slip gradients may play a role. It has long been known that proper mesh construction is critical in capturing incipient shear bands (Tvergaard et al., 1981). If the mesh is not aligned properly, the added mesh constraint can delay or prevent the appearance of a shear band. In the case of crystal modeling, the banded structure observed by Bhattacharyya et al., (2000) appeared in some grains and not in others, and the band features observed in Figure 16.8 were not present in another portion of the same grain, Figure 16.7. This indicates that these patterns can be excited or suppressed without significant modification to the stress field. Given that the local deformation field might not be difficult to perturb, it is possible that constraints imposed by the spatial discretization may be inhibiting gradients in the deformation field. Consider the orientation gradient shown in Figure 16.6. This gradient is oblique to the mesh, and it may not be possible for the elements to deform in a manner consistent with the observed orientation gradients. If this were the case, it may also explain the inability of the model to capture the correct sign for the slope on the left side of the specimen. 
The model assumes that the slip is homogeneous within an element, while experiments often show coarse slip traces. These present a challenge to the model in two ways. First is that the finite element model assumes displacement continuity. The assumption may be reasonable for the homogenized behavior of the element if there are a very large number of coarse slip bands within an element, but it is clearly violated when an element contains a countable number of coarse slip bands. The second issue brought about by coarse slip bands is that the dislocation structures formed on these coarse slip bands might present a more effective barrier to slip on other systems than is assumed in the models. Should coarse slip be considered specifically in the slip system hardening models?

Deficiencies in the slip system hardening relations used in crystal plasticity models are well known. A constant slip system interaction matrix was assumed. This does not properly account for interactions among slip systems (Bassani, 1990). Although using the more sophisticated slip system interaction model of Cuitino and Ortiz (1992) did not improve the predictions in the tricrystal simulations, that does not mean that the slip system model is unimportant. It is just not the dominant reason for the failure of the model in that simulation. It is also known that some crystals do not follow the Schmid law. Slip can depend on pressure as well as stress not acting directly on the slip system of interest (Qin and Bassani, 1992). This is generally not a big effect for aluminum, so it is doubtful that omission of non-Schmid contributions had a significant impact on these predictions.

\section{ACKNOWLEDGMENT}

Support of the experimental and computational work at the Alcoa Technical Center is gratefully acknowledged.

\section{REFERENCES}

Anand, L., and Kothari, M., 1996, A computational procedure for rate-independent crystal plasticity, $J$. Mech. Phys. Solids 44:525.

Asaro, R.J., 1983a, Crystal plasticity, J. Appl. Mech. 50:921.

Asaro, R.J., 1983b, Micromechanics of crystals and polycrystals, in: Advances in Applied Mechanics, Academic Press, New York.

Bassani, J.L., 1990, Single crystal hardening, Appl. Mech. Rev. 43:S320.

Bassani, J.L., and Wu, T.-Y., 1991, Latent hardening in single crystals II. Analytical characterization and predictions, Proc. Roy. Soc. Lond. A 435:21.

Beaudoin, A.J., Mather, K.K., Dawson, P.R., and Johnson, G.C., 1993, Three dimensional deformation process simulation with explicit use of polycrystalline plasticity models, Int. J. Plast. 9:833.

Beaudoin, A.J., Bryant, J.D., and Korzekwa, D.A., 1998, Analysis of ridging in aluminum, Metall. Trans. A 29:2323.

Becker, R., 1991, Analysis of texture evolution in channel die compression-I. Effects of grain interaction, Acta Metall. Mater. 39:1211.

Becker, R., 1992, Analysis of shear localization during bending of a polycrystalline sheet, J. Appl. Mech. 59:491.

Becker, R., 1998, Effects of strain localization on surface roughening during sheet forming, Acta Mater. 46:1385.

Becker, R., Butler, J.F., Hu, H., and Lalli, L.A., 1991, Analysis of an aluminum single crystal with unstable initial orientation (001)[110] in channel die compression, Metal. Trans. A 22:45.

Becker, R., and Panchanadeeswaran, S., 1995, Effects of grain interactions on deformation and local texture in polycrystals, Acta Metall. Mater. 43:2701. 
Bhattacharyya, A., El-Danaf, E., Kalidindi, S.R., and Doherty, R.D., 2000, Evolution of grain-scale microstructure during large strain simple compression of polycrystalline aluminum with quasicolumnar grains: OIM measurements and numerical simulations, Submitted for publication.

Bishop, J.F.W., and Hill, R., 1951, A theoretical derivation of the plastic properties of a polycrystalline face centered metal, Philos. Mag. 42:414.

Cuitino, A.M., and Ortiz, M., 1992, Computational modelling of single crystals, Modelling Simul. Mater. Sci. Engr. 1:225.

Harren, S.V., 2000, On the constitutive behavior of thermoelastic-viscoplastic crystals: theoretical and computational issues, Submitted for publication.

Harren, S.V., Dève, H.E., and Asaro, R.J., 1988, Shear band formation in plane strain compression, Acta Metall. 36:2435.

Havilcek, F., Kratochvil, J., Tokuda, M., and Lev, V., 1990, Finite element model of plastically deformed multicrystal, Int. J. Plast. 6:281.

Hill, R., and Rice, J.R., 1972, Constitutive analysis of elastic-plastic crystals at arbitrary strain, J. Mech. Phys. Solids 20:401.

Honneff, H., and Mecking, H., 1978, Textures in Metals, G. Gottstein and K. Lücke, eds., Springer, Berlin.

Humphreys, F.J., 1998, Quantitative metallography by electron backscattered diffraction, J. Microscopy 195:170.

Humphreys, F.J., 1999, Modelling microstructural evolution during annealing, Presented at workshop on Integrated Materials Modelling, Achen, Germany.

Hutchinson, J.W., 1964, Plastic deformation of B.C.C. polycrystals, J. Mech. Phys. Solids 12:25.

Kalidindi, S.R., Bronkhorst, C.A., and Anand, L., 1992, Crystallographic texture evolution in bulk deformation processing of FCC metals, J. Mech. Phys. Solids 40:537.

Kallivayalil, J.A., Weiland, H., and Becker, R., 1998, Unpublished research.

Kocks, U.F., Tomé, C., and Canova, G.R., 1986, Effective-cluster simulation of polycrystal plasticity, in: Large Deformations of Solids, J. Gittus, J. Zarka, and S. Nemat-Nasser, eds., Elsevier Applied Science, New York.

Miehe, C., 1996, Exponential map algorithm for stress updates in anisotropic multiplicative elastoplasticity for single crystals, Int. J. Num. Meth. Engr. 39:3367.

Mohan, R., Ortiz, M., and Shih, C.F., 1992, Mode mixity effects on crack tip deformation in ductile single crystals, Acta. Metall. Mater. 40:1907.

Ortiz, M., and Stainier, L., 1999, The variational formulation of viscoplastic constitutive updates, Comp. Meth. Appl. Mech. Engr. 171:419.

Panchanadeeswaran, S., Doherty, R.D., and Becker, R., 1996, Direct observation of orientation change by channel die compression of polycrystalline aluminum--use of a split sample, Acta Mater. 44:1233.

Peirce, D., Asaro, R.J., and Needleman, A., 1982, An analysis of nonuniform and localized deformation in ductile single crystals, Acta Metall. 30:1087.

Peirce, D., Asaro, R.J., and Needleman, A., 1983, Material rate dependence and localized deformation in crystalline solids, Acta Metall. 31:1951.

Polanyi, von M., 1922, Röntegenographische bestimmung von kristallanordnungen, Naturwissenschaften $10: 411$.

Qin, Q., and Bassani, J.L., 1992, Non-Schmid yield behavior in single crystals, J. Mech. Phys. Solids 40:813.

Radhakrishnan, B., and Baggathun, P., 2000, Manuscript in preparation.

Saeedvafa, M., and Rice, J.R., 1992, Crack tip fields in a material with three independent slip systems: NiAl single crystal, Modelling and Simul. Mater. Sci. Engr. i :53.

Schmid, E., 1924, Proc. Int. Cong. Appl. Mech. (Delft), 342.

Schwartz, A.J., Stölken, J.S., King, W.E., and Campbell, G.H., 2000, Lattice rotation during compression deformation of a [011] Ta Single Crystal, to appear in Mat. Sci Engr. A.

Stölken, J.S., King, W.E., Schwartz, A.J., Wall, M.A., and Nguyen, L., 1999, Reconstruction of a 3D microstructure using orientation imaging microscopy, in: Advances in Materials Problem Solving with the Electron Microscope, C. Allen, J. Bentley, U. Dahmen and I. Petrov, eds., MRS Proceedings, Boston.

Taylor, G.I., 1938, Plastic strain in metals, J. Inst. Met. 62:307.

Taylor, G.I., and Elam, C.F., 1925, The plastic extension and fracture of aluminum crystals, Proc. R. Soc. London, Sec A 108:28. 
Tvergaard, V., Needleman, A., and Lo, K.K., 1981, Flow localization in the plane strain tensile test, J. Mech. Phys. Solids 29:115.

Vogel, S., Klimanek, P., Juul Jensen, D., and Richter, H., 1996, Effect of texture on the development of grain size distribution during normal grain growth, Scripta Mater. 34:1225.

Weiland, H., and Becker, R., 1999, Analysis of mesoscale deformation structures in aluminum, Deformation induced Microstructures: Analysis and Relation to Properties, J.B. Bilde-Sørensen, J.V. Cartensen, N. Hansen, D. Juul Jensen, T. Leffers, W. Pantleon, O.B. Pedersen and G. Winther, eds., Risø National Laboratory, Roskilde, Denmark.

Zikry, M.A., and Nemat-Nasser, S., 1990, High strain-rate localization and failure of crystalline materials, $J$. Mech. Mater. 10:215. 